\title{
A PRELIMINARY APPROACH ON THE USE OF SATELLITE HYPERSPECTRAL DATA FOR GEOLOGICAL MAPPING
}

\author{
Nikolakopoulos G. K. ${ }^{1}$, Vaiopoulos D. A. ${ }^{2}$, and Skianis G. A. ${ }^{2}$ \\ ${ }^{1}$ I.G.M.E., Mesogion Str. 70, 115 27,Athens, knikolakopoulos@igme.gr \\ ${ }^{2}$ National and Kapodistrian University of Athens, Faculty of Geology and Geoenvironment, \\ Remote sensing Laboratory, vaiopoulos@geol.uoa.gr,skianis@geol.uoa.gr
}

\begin{abstract}
During the last decades remote sensing imagery has contributed significantly to mineral exploration. Motivated by the increasing importance of hyperspectral remote sensing, this study investigates the potential of the current-generation satellite hyperspectral data for geological mapping.

A narrow-band Hyperion image, acquired in summer 2001, was used. The study area is situated at Milos island.

Two different approaches were used for the reduction of the Hyperion bands. First, on the basis of histogram statistics the uncalibrated bands were selected and removed. Then the Minimum Noise Fraction was used to classify the bands according to their signal to noise ratio. The noisiest bands were removed and sixty bands were selected for further processing.

In order to make meaningful comparisons between image spectra and laboratory reflectance spetra, the image radiance values must be corrected (calibrated) to reflectance by removing the atmospheric effects. Atmospheric corrections techniques were applied to the selected Hyperion bands.

The comparison of the Hyperion hyperspectral data with the JPL spectral library gave quite encouraging results. Further processing of the data has to be done using the image analysis algorithms that have been developed specifically to exploit the extensive information contained in hyperspectral imagery.
\end{abstract}

Key words: Hyperion, Milos, Atmospheric corrections, Spectral libraries.

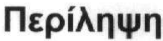

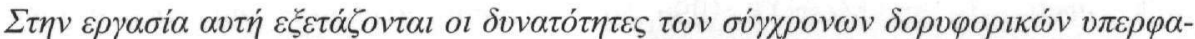

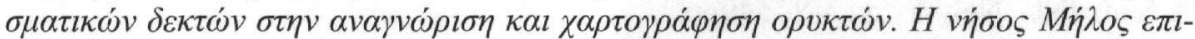

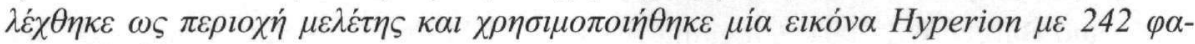
$\sigma \mu \alpha \tau \imath \kappa \varepsilon ́ \varsigma \zeta \omega ́ v \varepsilon \varsigma$.

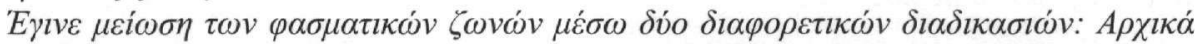

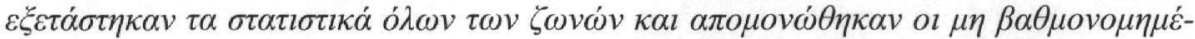

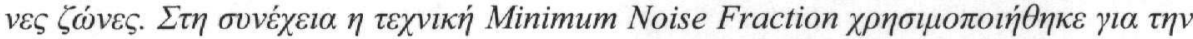

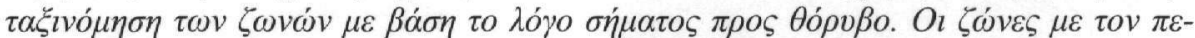

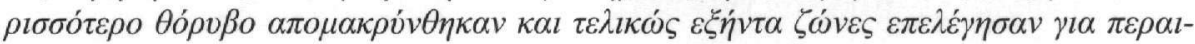
$\tau \varepsilon \dot{\varepsilon} \omega \epsilon \pi \varepsilon \xi \varepsilon \rho \gamma \alpha \sigma i \alpha$. 


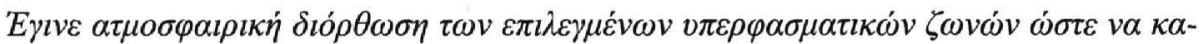

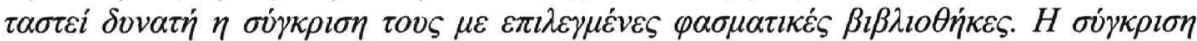

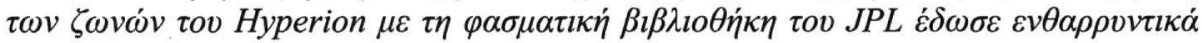
$\alpha \pi \circ \tau \varepsilon \lambda \dot{\varepsilon} \sigma \mu \alpha \tau \alpha$.

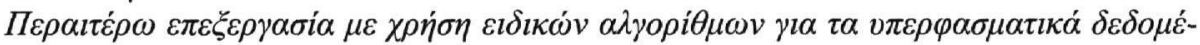

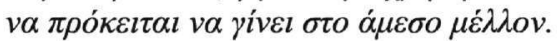

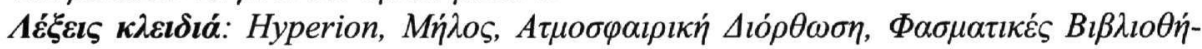
$\kappa \varepsilon \varsigma$.

\section{Introduction}

During the last decade one of the most significant progressions in remote sensing has been the development of hyperspectral sensors and the necessary software to analyze the resulting image data. Ten years ago, only spectral remote sensing experts had access to hyperspectral images and the necessary software tools to take advantage of them. The launch of satellite hyperspectral sensors such as Hyperion gave an impulse to the scientific community to use and validate the hyperspectral images for the earth surface mapping.

The widely used multispectral satellite remote sensing sensors, such as the Landsat (MSS, 4 bands), Landsat (TM \& ETM, 7 bands), SPOT (XS or XI, 3 \& 4 bands respectively) and ASTER (14 bands) drastically under-sample the information content available from a reflectance spectrum by making only a few measurements in spectral bands up to several hundred nanometers wide. Hyperspectral sensors, on the other hand, sample at close intervals (bands on the order of tens of nanometers wide) and have a sufficient number of spectral bands to allow construction of spectra that closely resemble those measured on laboratory instruments (Fig. 1). Such an image provides detailed spectral signatures for every pixel. These signatures often provide enough information to identify and quantify the material(s) existing within the pixels. A user could, for instance, employ a hyperspectral image to locate and quantify different types of minerals (kaolinite vs alunite) or vegetation species that might be present within an area of interest or even within a single pixel.

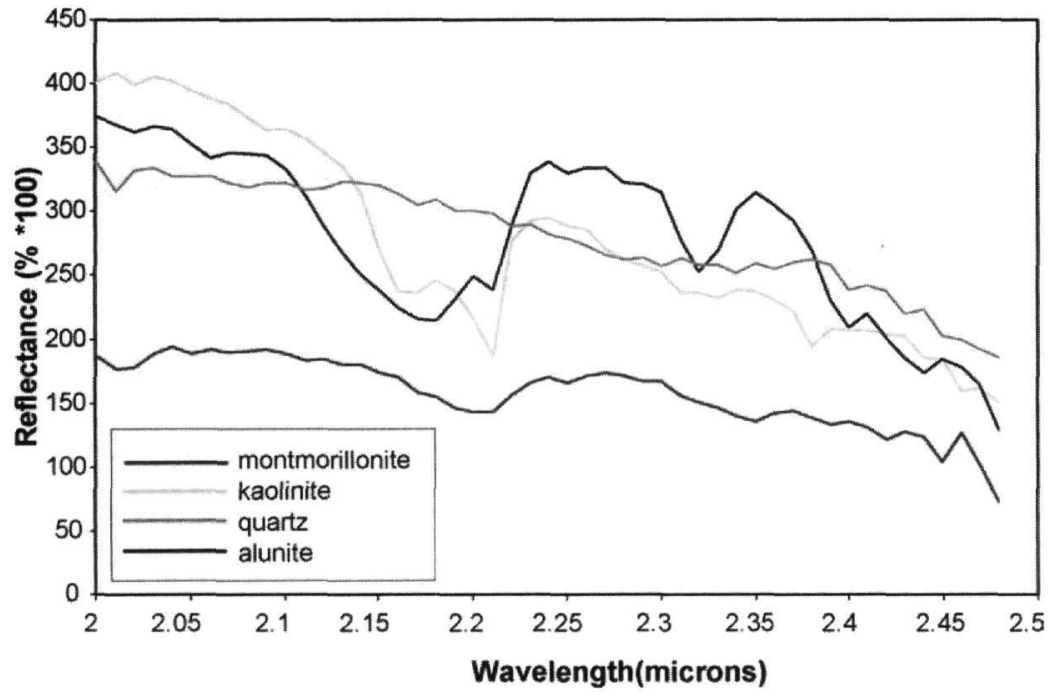

Figure 1 - Pixel spectra from an AVIRIS hyperspectral image (Van der Meer 2006)

Hyperspectral imaging systems are useful in identifying individual iron and clay minerals, which can provide details of hydrothermal alteration zoning (Sabins 1999) based on specific absorption features of these minerals. Various studies on the mapping of minerals using hyperspectral data can be found in (Crósta et al. 1998, Kruse and Boardman 1997, Rowan et al. 2000, Sabins 1999, Vaughan et al. 2003). Hyperspectral imagery has been used by geologists for mineral mapping 
(Hubbard et al. 2005, Debba et al. 2005) and to detect soil properties including moisture, organic content, and salinity (Ben-Dor et al. 2002). Vegetation scientists have successfully used hyperspectral data to identify vegetation species, mapping arid vegetation (Lewis et al. 2001) or compare vegetation indexes (Tomoaki et al. 2006, Haboudane et al. 2004). The hyperspectral imagery has also been used for mapping land and desertification (Shrestha et al. 2005).

Motivated by the increasing importance of hyperspectral remote sensing, this study investigates the potential of the current-generation satellite hyperspectral data for geological mapping.

The study area is Milos Island, in the Aegean Sea, Greece. Milos is, almost entirely, a volcanic island. It is estimated that volcanic activity in the island started 2-3 million years ago and ceased 90,000 years ago. Extinct volcanoes can still be seen at Firiplaka while the circular gulf of the island is considered to be a submerged caldera. There are also numerous locations around the island known for gas eruptions and hot springs suggesting volcanic activity. The western part of the island is covered by clay minerals. Every year, rich deposits of bentonite (used in drilling operations), kaolinite (ingredient of paper, rubber), perlite (for insulation) and pozzalana (cement industry) but also sulphur, barite and gypsum are exported. Many studies were presented for the Island of Milos. In one of the most recent (Ganas et al. 2002), airborne hyperspectral data (DAIS) were used for the detection and mapping of clay minerals. According to this study kaolinite (with various amounts of alounite and bentonite) has been shown to be widely dispersed over Western Milos. Small appearances of Hematite and Illite-jarosite have also been detected.

\section{Materials and Methods}

A narrow-band Hyperion image, acquired in summer 2001, was used. Hyperion is a pushbroom grating spectrometer ( 256 by 242 ) system with two spectrometers that share the same fore-optics (Liao et al. 2000). A VNIR CCD senses the first 70 bands and an HgCdTe SWIR detector senses bands 71-242. Not all bands are calibrated. The calibrated range extends from 430- $2400 \mathrm{~nm}$ and is comprised of 196 of the 242 bands, each with a nominal bandwidth of $10 \mathrm{~nm}$. Pre-launch SNR was measured at $60^{\circ}$ solar zenith for a $30 \%$ reflector with the results ranging from 161:1 for the visible to less than $40: 1$ for the SWIR (2100-2150 nm). The 256 spatial dimension of the detector array produces a $7.6 \mathrm{~km}$-wide ground swath with a pixel size of $29.88 \mathrm{~m}$. As the main objective of the study was to investigate the use of hyperspectral data for the geological mapping of Milos island, a subset of the image was used (Fig. 2).

\subsection{Atmospheric Correction}

Atmospheric scattering and absorption usually influence the intensity of the EM radiation that falls at the sensor of the remote sensing device. Targets of interest at ground surface may appear in the digital hyperspectral image with a considerably different spectral signature from that which would be obtained if atmospheric effects had not taken place. This may have negative consequences in interpreting remote sensing data, especially if the data have to be compared with digital spectral

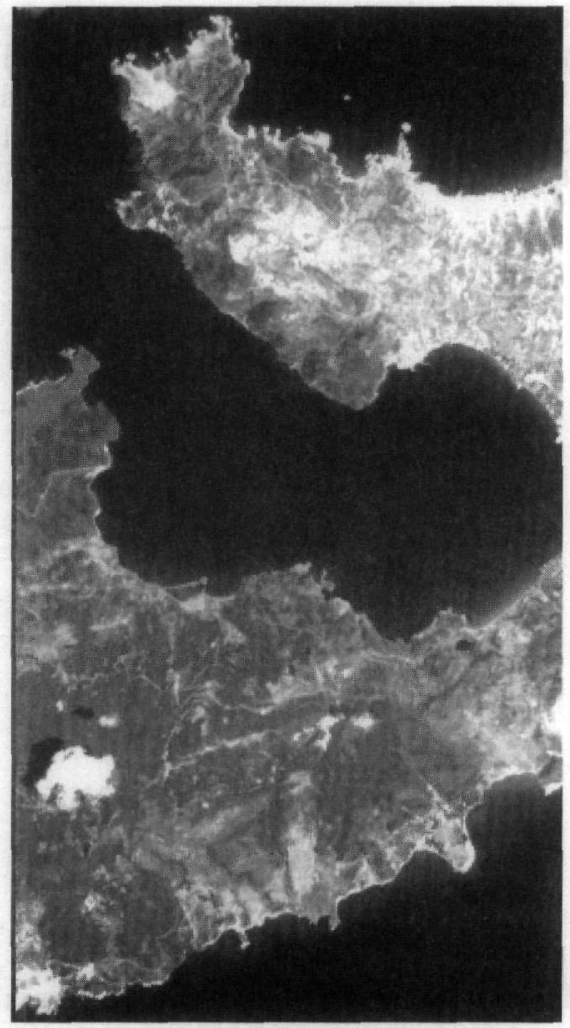

Figure 2 - A RGB image of Milos Island. Hyperion channels 215,45 \& 15 were used 
signatures collected in a lab.

Using ATCOR2/3 algorithms (Richter 1996a, Richter 1996b, Richter 1998) the Hyperion data were atmospherically corrected using a maritime mid-latitude profile. Two RGB combinations (45, 30, 15) of Milos island before (Fig. 3a) and after (Fig. 3b) the application of the atmospheric correction algorithm are presented below. There is a remarkable change of the colors of the atmospherically corrected image in comparison to the raw image.

\subsection{Band selection and endmember (targets) definition}

Hyperspectral data represents a challenge from a $\square$ data-processing point of view, as it can consist of hundreds of bands. A necessary first step is to reduce the complexity of the image by a dimensionality reduction, which compresses the image data to a few meaningful bands. Even the production of all the RGB band combinations is impossible for the Hyperion data as the number of the RGB band combinations is almost 14.000.000. Further, as we have already mentioned forty-six of the Hyperion bands are uncalibrated and could not be used in our study. In order to reduce the total number of the bands we proceed to a selection based on optical and statistical criteria.
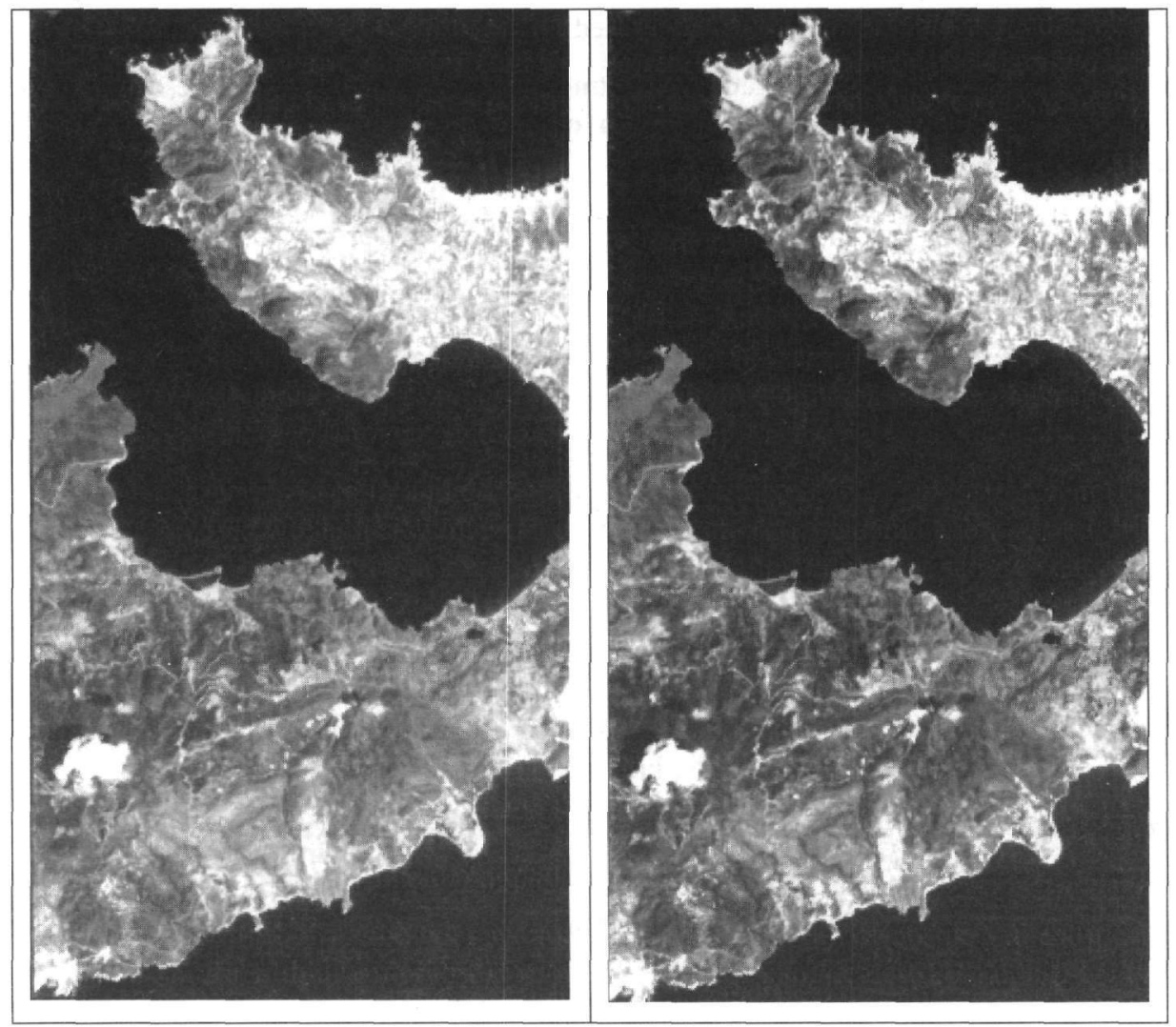

Figure 3 - a \& b. At the left the Hyperion raw image. At the right the Hyperion atmospherically corrected image. Hyperion channels $45,30 \& 15$ were used in both cases. There is a significant difference in the tonality

Firstly, all the 242 bands were examined visually. The optical control detected many bands dominated by noise and some other bands with no useful information (totally black or white images). Second, the histogram parameters of all the bands were examined. The statistical control of the minimum, maximum and standard deviation values confirmed the existence of bands corrupted by noise. As a result of the optical control only 152 bands were used for further processing. 
Then, the Minimum Noise Transform (Green et al. 1988), was employed to both remove noise and to compress the hyperspectral data into a smaller number of bands. The Minimum Noise Transform (MNF) transformation produces principal component-like images ordered according to increasing noise content. The first bands are information-dominated images in contrary to the last ones that are noise-dominated. The eigenvalues of the MNF bands are presented below (Fig. 4). Making several tests we have decided to use for the further processing the first sixty MNF bands.

Many image analysis algorithms have been developed specifically to exploit the extensive information contained in hyperspectral imagery. Spectral analysis methods usually compare pixel spectra with a reference spectrum (often called endmember or target). Target spectra can be derived from a variety of sources:

- Digital spectral library,

- In situ measurements using a spectro-radiometer

- Individual pixels of the image itself.

The first two procedures are used when the hyperspectral data are atmospherically corrected, the third one can be used when raw data are analyzed.

As the Hyperion data used in this study were atmospherically corrected but we didn't dispose any in situ measurements we decided to use two of the most common mineral spectral libraries (U.S.G.S. and JPL).

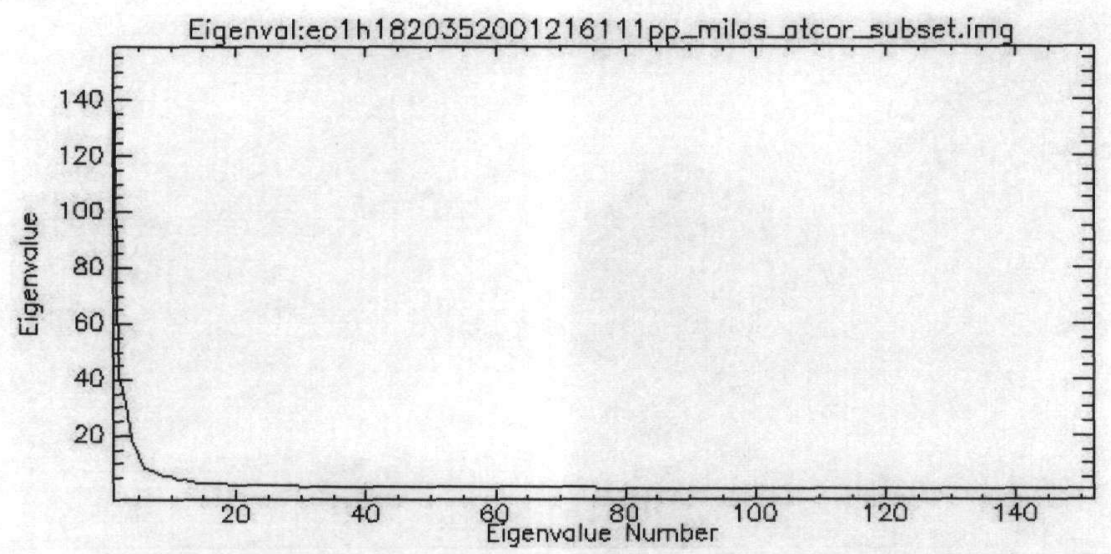

Figure 4 - The MNF bands eigenvalues. The first bands contain the biggest volume of information. The last ones are noise dominated

\subsection{Hyperspectral Data Analysis and Classification Theory}

The hyperspectral data analysis and classification techniques are divided in two major categories. In the first case we assume that every pixel contains only one target (endmember). This category includes standard supervised classifiers such as minimum distance or maximum likelihood (Richards et al. 1999), as well as tools developed specifically for hyperspectral imagery such as Spectral Angle Mapper (Yuhas et al. 1992) or Spectral Feature Fitting (Clark, et al.1991). The Spectral Angle Mapper (SAM) computes a spectral angle between each pixel spectrum and each target spectrum (Fig. 5). Small angle denotes big similarity between the pixel and target spectra. This spectral angle seems to be insensitive to changes in pixel illumination because increasing or decreasing illumination doesn't change the direction of the vector.

In Spectral Feature Fitting the user specifies a range of wavelengths within which a unique absorption feature exists for the chosen target. The pixel spectra are then compared with the target spectrum using two measurements. First, the depth of the feature in the pixel is compared with the 
depth of the feature in the target. Next, the shape of the feature in the pixel is compared with the shape of the feature In the target (often using a least-squares technique).

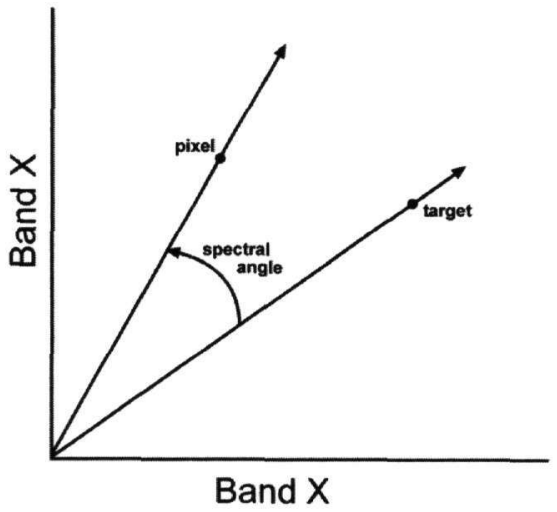

Figure 5 - Spectral Angle Mapper classification method. Pixel and target spectra plot as points in this scatter plot of pixel values. If a vector is drawn from the origin through each point, the angle between any two vectors constitutes the spectral angle between those two points

In the previous case we assumed that every pixel contains only one target (endmember). Unfortunately, natural surfaces are rarely composed of a single uniform material. These surfaces are more commonly made up of mixtures or assemblages of intimately mixed minerals, alteration products, or weathered constituents; and vegetation, water, and shadows. Spectral mixing is a consequence of the mixing of materials having different spectral properties within the ground field-of-view (GFOV) of a single image pixel (Fig. 6). This category includes classification methods such as Complete Linear Spectral Unmixing (Adams et al. 1986, Boardman 1989) or Matched Filtering (Boardman 1995). These techniques can detect quantities of a target that are much smaller than the pixel size itself. In cases of good spectral contrast between a target and its background, subpixel analysis could detect targets covering as little as 1-3 percent of the pixel.

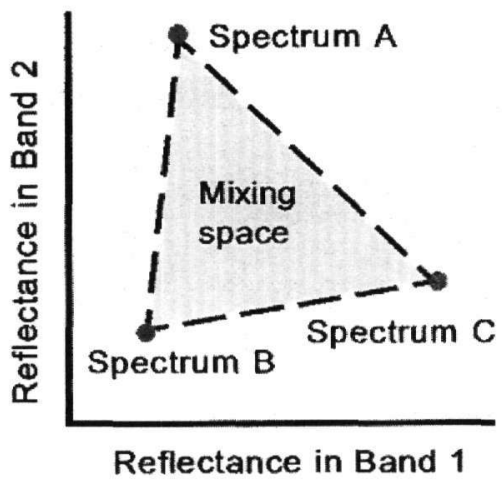

Figure 6 - N-dimensional plot of three target (endmember) spectra for a hypothetical 2-band case. All spectra that are mixtures of $A, B$ and $C$ alone must lie within the mixing space21

(TNTMIPS 2005)

The Complete Linear Spectral Unmixing assumes that the reflectance spectrum of any pixel is the result of linear combinations of the spectra of all endmembers inside that pixel. A linear combination in this context can be thought of as a weighted average, where each endmember's weight is directly proportional to the area of the pixel containing that endmember. If the spectra of all endmembers in the scene are known, then their abundances within each pixel can be calculated from each pixel's spectrum. The technique simply solves a set of $\mathrm{n}$ linear equations for each pixel, where 
$\mathrm{n}$ is the number of bands in the image. The unknown variables in these equations are the fractions of each endmember in the pixel. To have more equations than unknowns, it is necessary to have more bands than endmember materials. Hyperspectral imagery always covers this requirement.

Some hyperspectral image applications do not require finding the fractional abundance of all the targets (endmembers) in the scene. Instead the objective may be to detect the presence and abundance of a single target material. In this case a complete spectral unmixing is unnecessary. Each pixel can be treated as a potential mixture of the target spectral signature and a composite signature of all the other materials in the scene. Finding the abundance of the target component is then essentially a partial unmixing problem. Mixture-Tuned Matched Filtering (MTMF) algorithm filters the input image for good matches to the chosen target spectrum by maximizing the response of the target spectrum within the data and suppressing the response of everything else (Envi 2005).

Like complete unmixing, a pixel value in the output image is proportional to the fraction of the pixel that contains the target material. Any pixel with a value equal to zero or less would be interpreted as background. One potential problem with matched filtering is that it is possible to end up with false positive results. A solution is to calculate an additional measure called infeasibility. This measure is based on both noise and image statistics and indicates the degree to which the matched filter result is a feasible mixture of the target and the background. Pixels with high infeasibilities are likely to be false positive regardless of their matched filter value.

\section{Results and Discussion}

For the classification of the Hyperion data three of the above described algorithms were used. More specifically, the SAM, the Linear Spectral Unmixing, and the Mixture-Tuned Matched Filtering were applied to the sixty MNF bands (selected as described previously in paragraph 2.1). Several tests have been done using the spectral libraries provided form the USGS and the JPL. The spectral signatures of five clay minerals (alunite, illite, kaolinite, montmorilonite and pyrophillite) were selected from the two libraries and used for the classification.

The Spectral Angle Mapper classification technique gives better results in comparison to the other two techniques. Different clay minerals were detected and mapped. More especially the SAM algorithm detected at diverse sites alunite, illite, kaolinite, montmorilonite and pyrophillite. According to the bibliography (Ganas 2002) and to the 1/50.000 geological map those minerals are present in Milos Island. As expected the classification results varied depending:

- $\quad$ on the angle (Figs 7a, b) and

- $\quad$ on the spectral library used (Figs 8a, b)

With the use of JPL spectral library and a spectral angle value lower than twenty the alunite was not detected (Fig. 7a). When the spectral angle was raised to twenty-two small quantities of alunite were detected and mapped (Fig. 7b).

The role of the spectral library is also very important. When the USGS spectral library was used, only alunite, illite and montmorilonite minerals were detected (Fig. 8a). On the contrary the use of the JPL spectral library allowed the detection of two more clay minerals. More especially the kaolinite and the pyrophillite were also detected (Fig. 8b).

These preliminary results are in accordance with the results of a previous similar study that mapped a part of Milos Island using airborne DAIS data (Ganas et al. 2002). According to that study kaolinite, alounite, bentonite and small appearances of Hematite and Illite-jarosite have been detected. In our study the classification of satellite hyperspectral data with the use of JPL spectral library recognized five minerals (kaolinite, alunite, illite, montmorilonite and pyrophillite). 


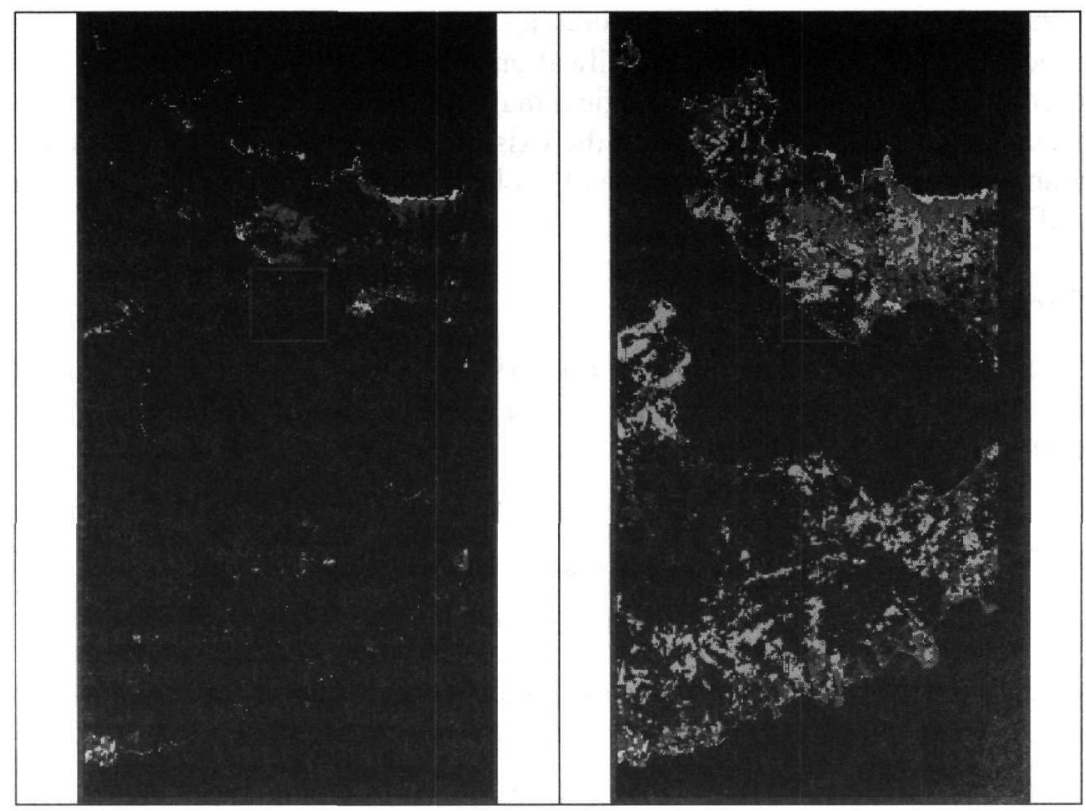

Figure 7 - a \& b. Classification results of the Hyperion data using the JPL spectral library and the Spectral Angle Mapper classification algorithm. The amount of the recognised clay minerals depends on the angle. At the left, the angle was set up to 20 degrees. At the right the angle was set up to $\mathbf{2 5}$ degrees. Allunite is presented with red colour, Illite with green, Kaolinite with blue, montmorilonite with yellow and Pyrophillite with cyan

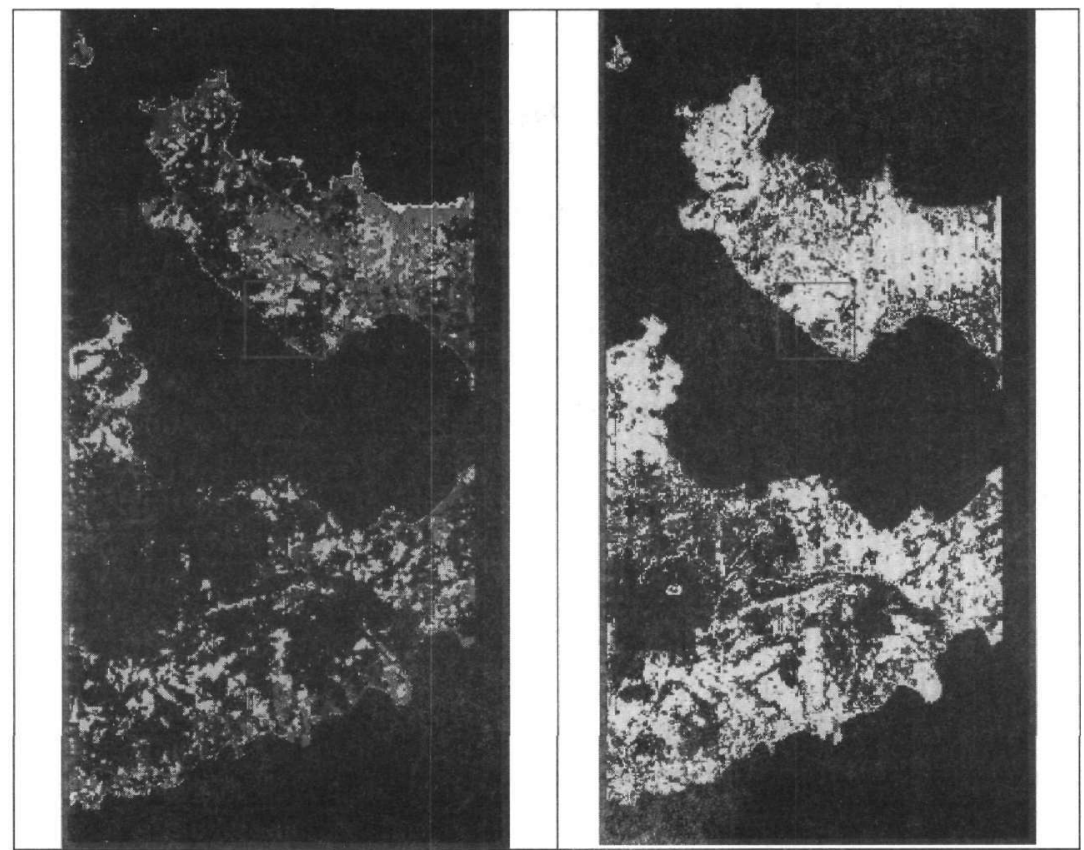

Figure 8 - a \& b. Classification results of the Hyperion data using the JPL spectral library at the left and the USGS spectral library at the right. The same Spectral Angle Mapper classification algorithm was used. When the USGS spectral library was used, only alunite and montmorilonite minerals were detected. Allunite is presented with red colour, Illite with green, Kaolinite with blue, montmorilonite with yellow and Pyrophillite with cyan 
Although these first results are quite encouraging it is recommended to proceed to a further analysis and processing of the data. The classification results should be controlled quantitatively and should be compared to the existing geological map of Milos. The exact location, shape and area of each appearance should be checked from the existing maps or should be verified in-situ. Furthermore, an analysis of the Hyperion data and a classification with endmembers derived from the image itself should also be done.

\section{References}

Adams, J. B., Smith, M. O., and Johnson, P.E., 1986. Spectral mixture modelling: A new analysis of rock and soil types at the Viking Lander 1 site, Journal of Geophysical Research, 91(B8), 8090-8112.

Ben-Dor, E., Patkin, K., Banin, A., and Karnieli, A., 2002. Mapping of several soil properties using DAIS-7915 hyperspectral scanner data - a case study over soils in Israel, International Journal of Remote Sensing 23 (6), 1043-1062.

Boardman, J. W., 1989. Inversion of imaging spectrometry data using singular value decomposition, Proceedings of the Twelfth Canadian Symposium on Remote Sensing, 4., 2069-2072.

Boardman, J. W., Kruse, F. A., and Green, R. O., 1995. Mapping target signatures via partial unmixing of AVIRIS data". In Summaries of the Fifth JPL Airborne Earth Science Workshop, JPL Publication 95-1, v. 1, 23-26pp.

Clark, R. N., Swayze, G. A., Gallagher, A., Gorelick, N., and Kruse, F. A., 1991. Mapping with imaging spectrometer data using the complete band shape leastsquares algorithm simultaneously fit to multiple spectral features from multiple materials. In Proceedings of the Third Airborne Visible/Infrared Imaging Spectrometer (AVIRIS) workshop, JPL Publication 9128, 2-3pp.

Crósta, A. P., Sabine, C., \& Taranik, J. V., 1998. Hydrothermal alteration mapping at Bodie, California, using AVIRIS hyperspectral data, Remote Sensing of Environment, 65(3), 309- 319.

Debba, P., van Ruitenbeek, F.J.A., van der Meer, F.D., Carranza, E.J.M., and Stein, A., 2005. Optimal field sampling for targeting minerals using hyperspectral data, Remote Sensing of Environment 99 (2005) $373-386$.

Envi, 2005. Envi User's Manual, Research Systems Inc, Pearl East Circle.

Ganas, Ath., and Ferrier, Graham, 2002. Mapping of diagnostic clay mineralsin epithermal gold deposits of Milos Island (Greece) using hyperspectral data from the DAIS airborne radiometer, 6th Pan-Hellenic Geographical Congress, Thessaloniki, Volume II, 118 - 126pp.

Green, A.A, Berman, M., Switzer, P., and Craig, M.D., 1988. A transformation for ordering multispectral data in terms of image quality with implications for noise removal, IEEE Transactions on geoscience and remote sensing, 26, 65-74.

Haboudane, Dr, Miller, J.R., Pattey, E., Zarco-Tejada, P.J., and Strachan, I.B., 2004. Hyperspectral vegetation indices and novel algorithms for predicting green LAI of crop canopies: Modeling and validation in the context of precision agriculture, Remote Sensing of Environment 90 (2004) 337-352.

Hubbard E.B., and Crowley, J.K., 2005. Mineral mapping on the Chilean-Bolivian Altiplano using co-orbital ALI, ASTER and Hyperion imagery: Data dimensionality issues and solutions, Remote Sensing of Environment 99 (2005), 173 - 186. 
Kruse, F. A., and Boardman, J. W., 1997. Characterization and mapping of kimberlites and related diatremes in Utah, Colorodo, and Wyoming, USA, using the airborne visible/infrared imaging spectrometer (AVIRIS), ERIM Proceedings of the 12th international conference on applied geologic remote sensing, 21-28pp.

Liao, L., Jarecke, P., Gleichauf, D., and Hedman, T., 2000. Performance Characterization of the Hyperion Imaging Spectrometer Instrument, Proc. of SPIE, 4101, 22-26.

Lewis, M., Jooste, V., and de Gasparis, A.A., 2001. Discrimination of Arid Vegetation with Airborne Multispectral Scanner Hyperspectral Imagery, IEEE Transactions On Geoscience And Remote Sensing, 39(7), 1471-1479.

Richards, J.A., and Jia, X., 1999. Remote Sensing Digital Image Analysis, an Introduction, Third Edition. Springer-Verlag: Berlin.

Richter, R., 1996. “A spatially adaptive fast atmospheric correction algorithm” Int. J. Remote Sensing, 17, 1201-1214.

Richter, R., 1996. "Atmospheric correction of satellite data with haze removal including a haze/clear transition region", Computers \& Geosciences, 22, 675-681.

Richter R., 1998. "Correction of satellite imagery over mountainous terrain”, Applied Optics, 37, 4004-4015.

Rowan, L. C., Crowley, J. K., Schmidt, R. G., Ager, C. M., and Mars, J. C. 2000. Mapping hydrothermally altered rocks by analyzing hyperspectral image (AVIRIS) data of forested areas in the Southeastern United States, Journal of Geochemical Exploration, 68(3), 145- 166.

Sabins, F. F., 1999. Remote sensing for mineral exploration, Ore Geology Reviews, 14 (Issues 3 4), $157-183$.

Shrestha, D.P., Margate, D.E., van der Meer, F., and Anh, H.V., 2005. Analysis and classification of hyperspectral data for mapping land degradation: An application in southern Spain, International Journal of Applied Earth Observation and Geoinformation, 7 (2005), 85-96.

TNTMIPS, 2005. Introduction to Hyperspectral Imaging, Microimages, Inc. Lincoln, Nebraska, USA, 24pp.

Tomoaki M., Huete A., and Yoshioka, H., 2006. An empirical investigation of cross-sensor relationships of NDVI and red/near-infrared reflectance using EO-1 Hyperion data, Remote Sensing of Environment 100, 223 - 236.

Van der Meer, Freek, 2006. The effectiveness of spectral similarity measures for the analysis of hyperspectral imagery, International Journal of Applied Earth Observation and Geoinformation, 8 (2006), 3-17.

Vaughan, R. G., Calvin, W. M., and Taranik, J. V., 2003. SEBASS hyperspectral thermal infrared data: Surface emissivity measurement and mineral mapping, Remote Sensing of Environment, 85(1), 48-63.

Yuhas, R.H., Goetz, A. F. H., and Boardman, J. W., 1992. Discrimination among semiarid landscape endmembers using the spectral angle mapper (SAM) algorithm. In Summaries of the Third Annual JPL Airborne Geoscience Workshop, JPL Publication 92-14, vol. 1, 147 149pp. 